COMMUNICATIONS IN

ANALYSIS AND GEOMETRY

Volume 13, Number 4, 801-820, 2005

\title{
Diagrams and the second homotopy group
}

\author{
Max Forester ${ }^{1}$ And Colin Rourke
}

\begin{abstract}
We use Klyachko's methods $[4,6,7,10]$ to prove that, if a 1-cell and a 2-cell are added to a complex with torsion-free fundamental group, and with the 2 -cell attached by an amenable $t$-shape, then $\pi_{2}$ changes by extension of scalars. It then follows using a result of [2] that the resulting fundamental group is also torsion free. We also prove that the normal closure of the attaching word contains no words of smaller complexity.
\end{abstract}

\section{Introduction.}

We consider the following problem. Suppose that $K$ is a CW complex and that $L$ is formed from $K$ by adding cells of dimension $\leq 2$.

Problem. Describe $\pi_{2}(L)$ in terms of $\pi_{2}(K)$.

The problem is interesting in its own right, but also because of its relation to Whitehead's conjecture [15] (still unsolved). This says that if $K$ is 2dimensional with non-zero $\pi_{2}$, then $L$ also has non-zero $\pi_{2}$.

We shall answer the question completely in a special case. Suppose that $L=K \cup e^{1} \cup e^{2}$ and let $t$ be the new generator of $\pi_{1}$ determined by $e^{1}$. Then, the attaching map for $e^{2}$ represents a word $w$ in $G *\langle t\rangle$ where $G=\pi_{1}(K)$ which we can assume to be cyclically reduced. The $t$-shape of $w$ is the unreduced word formed by occurrences of $t$. There is a useful notion of amenable t-shape that was introduced in [6]. This includes all $t$-shapes having total exponent \pm 1 in $t$. The exact definition will be recalled in Section 1.

Main Theorem. Suppose that $L=K \cup e^{1} \cup e^{2}$ and that the attaching map for $e^{2}$ represents $w$ in $G *\langle t\rangle$ where $G=\pi_{1}(K)$ and $t$ is the new generator of $\pi_{1}$ determined by $e^{1}$. Suppose

(1) $G$ is torsion-free

(2) the t-shape of $w$ is amenable.

\footnotetext{
${ }^{1}$ Research supported by EPSRC grant GR/N20867
} 
Then, there is an isomorphism of $\mathbb{Z} \pi_{1}(L)$-modules

$$
\pi_{2}(L) \cong \mathbb{Z} \pi_{1}(L) \otimes_{\mathbb{Z} \pi_{1}(K)} \pi_{2}(K)
$$

This shows that in passing from $K$ to $L, \pi_{2}$ changes in the simplest possible way, i.e. by "extension of scalars". No elements are killed, and all new elements are accounted for by the change in fundmental group.

Note that under conditions (1) and (2), Fenn and Rourke have solved the adjunction problem, and proved that $\pi_{1}(K) \rightarrow \pi_{1}(L)$ is injective [6]. From this, it follows easily that if $K$ is 2-dimensional, then $\pi_{2}(K) \rightarrow \pi_{2}(L)$ is also injective. (Let $\widetilde{K}, \widetilde{L}$ be the universal covers; then $K \subset L$ lifts to $\widetilde{K} \subset \widetilde{L}$ and hence $\pi_{2}(K) \cong H_{2}(\widetilde{K}) \subset H_{2}(\widetilde{L}) \cong \pi_{2}(L)$, where the middle inclusion follows from the fact that $\widetilde{L}-\widetilde{K}$ is 2-dimensional.) So, this paper gives no new information for the Whitehead conjecture itself. However, note that we do not need to assume that $K$ is 2-dimensional for our results.

The key observation of this paper is that the Klyachko proof used by Fenn and Rourke actually shows much more than is needed for the adjunction problem. For the latter, one needs that there are no diagrams based on $w$ with a non-trivial boundary. The proof actually shows that there are no irreducible diagrams whatever the boundary. This translates into the statement that $\pi_{2}\left(K^{+}, K\right)$ maps onto $\pi_{2}(L, K)$ where $K^{+}=K \cup e^{1}$. From this, the Main Theorem follows by standard algebraic topology.

It is interesting to note that, whilst the adjunction problem is still open for torsion-free groups, and indeed for general groups provided the exponent sum of $t$ in $w$ is non-zero, the Main Theorem fails if either hypothesis (1) or (2) is dropped. We shall see that in either case, there are both irreducible diagrams and elements of $\pi_{2}(L, K)$ not accessible from $\pi_{2}\left(K^{+}, K\right)$. This has consequences for a possible proof of the adjunction problem. Fenn and Rourke [6, p. 70] suggest that the Klyachko methods should be strong enough to prove the 1-generator 1-relator adjunction problem for torsion-free groups for any $t$-shape. While this may be true, this paper shows that the methods will need to be extended considerably if they are to work for a $t$-shape which is a proper power.

This paper provides an alternate approach to part of the Klyachko method, namely the "algebraic trick" described in [6, pp. 64-66]. Here, this step is explained in terms of diagrams; see, in particular, Figure 6.

The diagram methods also apply to the Cohen-Rourke results [4] and imply strong information about the normal closure $\langle\langle w\rangle\rangle$ of $w$ in $G *\langle t\rangle$ : no word of complexity smaller than the complexity of $w$ lies in $\langle\langle w\rangle\rangle$ (complexity is defined in Section 1). It should be noted that this last result 
can be deduced directly from the Cohen-Rourke methods without using the methods of the present paper.

The basic geometric result, that there are no irreducible diagrams, is related to the notion of asphericity of relative presentations introduced in [2] and also studied in [5, 1, 9, 12]. However, we use a slightly different notion of irreducibility, involving basepoints. This is to ensure that "dipoles" can always be removed by a homotopy. The difference between the two notions is readily apparent in the second example of Section 4.

On the other hand, the two notions differ only in the case of words that admit a non-trivial symmetry (considered as cyclic words), and such words are outside the scope of the Main Theorem. Hence, the basic diagram result may be interpreted as saying that relative group presentations satisfying conditions (1) and (2) are aspherical in the sense of [2].

Combining our result with one of the main results of [2], which states (in our case) that any finite subgroup of $\pi_{1}(L)$ is conjugate to a subgroup of $\pi_{1}(K)$, it follows that $\pi_{1}(L)$ is also torsion free, which answers a question of Cohen and Rourke [4]. In a future paper, ${ }^{2}$ we shall give a direct proof of this result and deduce consequences for the multivariable adjunction problem. We would like to thank the referee for pointing out the connection of our result with the notion of aspherical relative presentations, which has made this important consequence clear to us.

Here is an outline of the paper. In Section 1, we define diagrams and irreducibility and state our main results about the non-existence of diagrams. Section 2 contains the proofs of the diagram theorems and in Section 3, we translate the diagram results into statements about $\pi_{2}$ and prove the Main Theorem. In Section 4, we give the counterexamples mentioned above and discuss the limits of the Klyachko methods for proving the adjunction problem over torsion-free groups. In Section 5, we give a generalisation of the Diagram Theorem which implies the complexity result mentioned above.

\section{Diagrams.}

Let $G$ be a group and $w \in G *\langle t\rangle$. By a disc $D$ labelled by $w$, we mean a 2-disc with legs, one for each occurrence of $t$ or $t^{-1}$ in $w$. The legs are "thick arcs", in other words, trivial bundles with fibre $I$. Further, the fibres are oriented and labelled $t$ in such a way that the $t$-shape of $w$ can be read from the legs by reading around the the boundary in an anti-clockwise direction

\footnotetext{
${ }^{2}$ Now published as "The adjunction problem over torsion-free groups" PNAS 102 (2005) 12670-12671
} 
and reading $t$ if we cross a leg whose fibres are oriented in this direction and $t^{-1}$ otherwise. Further, the segments of the boundary of $D$ between the legs are labelled by elements of $G$ and the whole of $w$ is recovered by reading these labels and the legs. Finally, we fix a basepoint in the word $w$ (such as at the beginning) and indicate this on the boundary of the $w$-disc.

A disc labelled by $\bar{w}$ means the reflection of a disc labelled by $w$. A $w$ diagram means a finite collection of discs in the plane labelled by $w$ and $\bar{w}$ together with thick arcs with oriented fibres labelled $t$ (called $t$-arcs) which complete the legs compatibly with the orientations. Fibred annuli ( $t$-circles) are also allowed. The diagram cuts the plane into a number of finite regions (the "inside regions") and one infinite region, the "outside".

Each region of the diagram determines a word in $G$ by reading anticlockwise around the region for inside regions and clockwise for the outside region using the labels on the adjacent discs. We require that this word is the identity in $G$ for each inside region. An example is shown in Figure 1. In this example, $w=\operatorname{tatbt}^{-1} c$ and $G$ satisfies the relations $b^{2}=c^{2}=1$, $a^{-1} b a=c$.

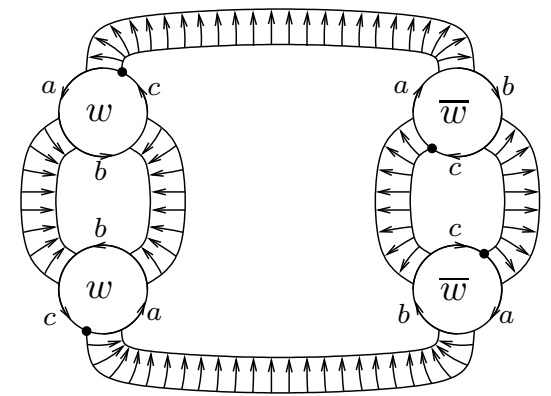

Figure 1: A $w$-diagram with $w=t_{a t b t^{-1} c}$

A diagram is irreducible if:

(1) it is connected,

(2) it contains at least one disc labelled by $w$ or $\bar{w}$,

(3) it does not contain pairs of discs of the type illustrated in Figure 2, where the joining 'leg' represents the same occurrence of $t$ or $t^{-1}$ in $w$ and $\bar{w}$.

Condition (2) means that the diagram does not just consist of one $t$ circle. Condition (3) needs care if $w$ has symmetry. The "same occurrence 


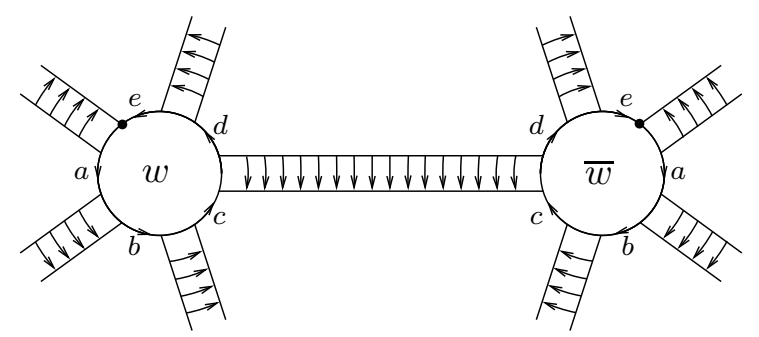

Figure 2: Disc pair with $w=t^{-1} a t b t c t^{-1} d t e$

of $t$ or $t^{-1}$ " means the same position with respect to the basepoint.

The diagram shown in Figure 1 is irreducible.

We now recall the definition of amenable $t$-shapes from $[6,7]$. Note that $t$-shapes are always considered as cyclic words.

Write a given $t$-shape $T$ as an unreduced cyclic word in the symbols $t$ and $t^{-1}$. The Magnus derivative $D(T)$ is the $t$-shape given by deleting all subwords $t t^{-1}$ and closing up. A more symmetrical definition is given in [7, p. 161]. It is easy to see that $D^{m}(T)=t^{q}$ for some $q \in \mathbb{Z}$ for $m$ sufficiently large. Call $\min \left\{m \mid D^{m}(T)=t^{q}\right\}$ the complexity of $T$ and call $D^{m-1}(T)$ the root of $T$. A clump in a $t$-shape is a maximal connected subsequence of the form $t^{q}$ or $t^{-q}$ where $q>1$. A one-clump shape is a $t$-shape which has exactly one clump which is not the whole shape. In other words, it is a shape of the form $t^{p}\left(t t^{-1}\right)^{q}$ for $p, q>0$ or its inverse. A $t$-shape is amenable if it has root either $t t^{-1}$ or a one-clump shape. For future reference, we shall call these shapes amenable root shapes.

It is easy to see that any $t$-shape of total exponent sum 1 in $t$ is amenable since the root must be of the form $t\left(t t^{-1}\right)^{q}$ for $q>0$. On the other hand, no $t$-shape which is a proper power is amenable.

Diagram Theorem. Suppose that $w$ is a word in $G *\langle t\rangle$ with an amenable $t$-shape and that $G$ is a torsion-free group. Then, there are no irreducible $w$-diagrams.

The proof is really an observation. This is what the Klyachko proof of the Kervaire conjecture (and the extension given by Fenn-Rourke in $[6,7]$ ) actually shows. We shall need to go carefully through the details to make this clear and we shall do this in the next section.

If $G$ is not torsion-free, then there are many easily constructed irreducible diagrams. In [13], it is shown how to construct infinite families of irreducible diagrams made of "units". Figure 1 is a simple example of this construction: 
it is made of two 2-units. All diagrams made in this way require the group to have torsion. Short [14] has investigated diagrams made of units in detail and proved that none gives a counterexample to the Kervaire conjecture.

If $G$ is torsion-free, but the word is not amenable, then again irreducible diagrams may exist. Explicit examples are given in Section 4.

\section{Proof of the Diagram Theorem.}

We follow the general pattern of proof of $[10]$ as exposited in $[4,6]$. We prove first a special case, when the $t$-shape is an amenable root shape (either $t t^{-1}$ or a one-clump shape) and then use the "algebraic trick" described in $[6, \mathrm{p}$. 64-66] to convert this to the general case.

We need to generalise diagrams for a set of words in $\Gamma *\langle t\rangle$. The Root Shape Theorem below yields the special case of the Diagram Theorem by taking $\Gamma=G$. In the proof of the general case, $\Gamma$ will represent $G *\langle s\rangle$.

Let $\Gamma$ be a group and $w_{0} \in \Gamma *\langle t\rangle$ be cyclically reduced. An element of $\Gamma$ in $w_{0}$ sandwiched cyclically between an occurrence of $t$ and $t^{-1}$ is called a top coefficient of $w_{0}$. One sandwiched between an occurrence of $t^{-1}$ and $t$ is called a bottom coefficient. The others are called middle coefficients.

Now, let $H$ be a subgroup of $\Gamma$ and $g$ an element of $\Gamma$. We say that $g$ is free relative to $H$ if the subgroup $\langle g, H\rangle$ of $\Gamma$ generated by $g$ and $H$ is naturally the free product $\langle g\rangle * H$ of an infinite cyclic group $\langle g\rangle$ with $H$.

We shall use the following working hypotheses.

\subsection{Working hypotheses.}

Suppose that $H$ and $H^{\prime}$ are two isomorphic subgroups of a group $\Gamma$ under the isomorphism $h \rightarrow h^{\phi}, h \in H$.

Let $w_{0}$ be a word in $\Gamma *\langle t\rangle$ with amenable root $t$-shape and let $a_{i}, b_{i} \in \Gamma$ be the bottom and top coefficients of $w_{0}$ respectively listed in any order. Suppose that for each $i, a_{i}$ is free relative to $H$ and $b_{i}$ is free relative to $H^{\prime}$. Let $W \subset \Gamma *\langle t\rangle$ be the set of words $\left\{w_{0}, h^{t}\left(h^{\phi}\right)^{-1} \mid h \in H\right\}$ where $h^{t}=t^{-1} h t$.

Definitions. A $W$-diagram is a diagram with discs labelled by elements of $W$ or their inverses (denoted $\bar{w}_{0}$ etc) with the same interpretation as in Section 1, together with arcs completing the legs and such that words read around the boundaries of inside regions are the identity in $\Gamma$, as in the definition of a $w$-diagram.

Notice that a disc labelled by $h^{t}\left(h^{\phi}\right)^{-1}$ has just two legs emerging and 
the boundary arcs between the legs are labelled $h$ and $h^{\phi}$; see Figure 3 .

A $W$-diagram is irreducible if:

(1) it is connected,

(2) it contains at least one disc labelled by $w_{0}$ or $\bar{w}_{0}$,

(3) it does not contain pairs of discs of the type illustrated in Figure 2, where the joining 'leg' represents the same occurrence of $t$ or $t^{-1}$ in $w_{0}$ and $\bar{w}_{0}$,

(4) it does not contain a string of two-leg discs labelled in order by $h_{i}^{t}\left(h_{i}^{\phi}\right)^{-1}, i=1,2, \ldots, p$ where $h_{1} h_{2} \ldots h_{p}=1$; see Figure 3.

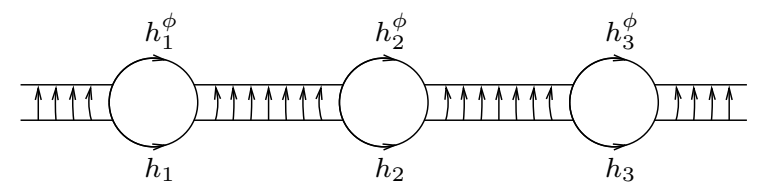

Figure 3: A string of two-leg discs

Root Shape Theorem. Assume the working hypotheses. Then, there are no irreducible $W$-diagrams.

Note that by taking $H$ and $H^{\prime}$ to be trivial in the theorem, we can deduce a special case of the Diagram Theorem, namely the case when the $t$-shape is an amenable root shape.

Proof. Let $D$ be an irreducible $W$-diagram. We convert $D$ into a cell subdivision of the 2-sphere, which is essentially the dual diagram, by putting a vertex in each region and joining by an edge across each $t$-arc; see [4, Figure 5]. We now define a traffic flow as described on page 68 of [6] and obtain a contradiction in exactly the same way as in [4] or [6]. The flow is described for suitable $t$-shapes which are more general than one-clump shapes but do not include the shape $t t^{-1}$, but this shape has a very easy flow. All cells are bigons and a traffic flow (without stops) is defined as for the bigons on the right of [6, Figure 5]. The details for the contradiction are clearest in [4, p. 137]. Crashes can only occur at vertices. There may be a crash at the vertex in the outside region of $D$. But since there are at least two crashes in different places, there must be a crash at an internal vertex. But the flow has been chosen so that, at an internal vertex where all the cars come 
together at the same time, the labels around the corners are all $\left\{a, a^{-1}\right\}$ for some coefficient $a=a_{i}$ of $w_{0}$ together with elements of $H$ or $\left\{b, b^{-1}\right\}$ for some coefficient $b=b_{i}$ of $w_{0}$ together with elements of $H^{\prime}$. For definiteness, assume that we are in the former situation. Then, we can read an (unreduced) word of the form $a^{\epsilon_{1}} h_{1} h_{2} \ldots h_{i_{1}} a^{\epsilon_{2}} h_{1} h_{2} \ldots h_{i_{2}} a^{\epsilon_{3}} \ldots$ which is 1 in $\Gamma$. Now, if this word contains a subword of the form $a^{\epsilon} a^{-\epsilon}$ then $D$ fails part (3) of the definition of irreducibility and if it contains a subword of the form $h_{1} h_{2} \ldots h_{i}$ which is 1 in $\Gamma$, then it fails part (4). Since $D$ is irreducible, neither of these happen and the word gives a non-trivial relation in $\langle a, H\rangle$, contradicting the assumption that $a$ is free relative to $H$.

\subsection{Proof of the Diagram Theorem.}

We now convert the Root Shape Theorem into the Diagram Theorem by using the "algebraic trick" of Klyachko described in [6, pp. 64-66] and [4, pp. 137-139]. However, we need to do this on the level of diagrams. Recall the following definitions:

Consider the exponent sum homomorphism ex: $G *\langle s\rangle \rightarrow \mathbb{Z}$. It is well known that $K$, the kernel of ex, is a free product of copies of $G$ generated by elements of the form $g^{s^{\mathcal{O}}}=s^{-\mathcal{O}} g s^{\mathcal{O}}, 1 \neq g \in G$.

Any element of $K$ has a canonical expression of the form $k=g_{1}^{s^{\mathcal{O}_{1}}} \cdots g_{r}^{s^{\mathcal{O}_{r}}}$, where $\mathcal{O}_{i} \neq \mathcal{O}_{i+1}$ for each $i$. We shall call the $g_{i}^{s^{\mathcal{O}_{i}}}$ the canonical elements of $k$. Let $\min (k)$ be the minimum value of $\mathcal{O}_{i}, i=1, \ldots, r$ and $\max (k)$ the maximum value. Fix a positive integer $m$. Consider the following subgroups of $K$ :

$$
\begin{aligned}
H & =\langle k \in K \mid \min (k) \geq 0, \max (k) \leq m-2\rangle \\
H^{\prime} & =\langle k \in K \mid \min (k) \geq 1, \max (k) \leq m-1\rangle \\
J & =\langle k \in K \mid \min (k) \geq 0, \max (k) \leq m-1\rangle
\end{aligned}
$$

and the following subsets:

$$
\begin{aligned}
& X=\{k \in K \mid \min (k)=0, \max (k) \leq m-1\} \\
& Y=\{k \in K \mid \min (k) \geq 0, \max (k)=m-1\} .
\end{aligned}
$$

We consider words in $(G *\langle s\rangle) *\langle t\rangle$ obtained from an amenable root $t$ shape by "blowing up". To be precise, we cyclically insert an element of $X$ (respectively $Y, J$ ) in each top (respectively bottom, middle) position, i.e. between successive occurrences of $t$ and $t^{-1}$ (respectively $t^{-1}$ and $t, t$ and $t$ or $t^{-1}$ and $\left.t^{-1}\right)$. 
Now, take the resulting word $w(s, t)$, substitute $t$ for $s$, and reduce cyclically to obtain $w$. The $t$-shape of any $w$ obtained in this way is amenable of complexity $\mathrm{m}$. See [7] for the details of the proof that this coincides with the definition given earlier and the (apparently more general) one given in [6]. The root shape of $w$ is the original $t$-shape before blowing up. We call $w(s, t)$ the blown up form of $w$ (also called normal form in $[6,7]$ ). Write it as

$$
w(s, t)=t^{\epsilon_{1}} x_{1}(s) t^{\epsilon_{2}} x_{2}(s) \ldots t^{\epsilon_{n}} x_{n}(s)
$$

where each $x_{i}(s)$ belongs to the appropriate subset $X, Y$ or $J$.

We now describe a procedure for converting irreducible $w$-diagrams to irreducible $W$-diagrams. Here, $W=\left\{w(s, t), h^{t}\left(h^{\phi}\right)^{-1} \mid h \in H\right\}$ where $\phi: H \rightarrow H^{\prime}$ is given by $h^{\phi}=h^{s}$, and $w(s, t)$ is regarded as a word with coefficients in $\Gamma=G *\langle s\rangle$ and having amenable root $t$-shape. The process is illustrated in Figures 4-6 for the amenable word $w=a t t b t^{-1} t^{-1} c d t^{-1} e t^{2}$.

Given a $w$-diagram, first, we replace every $w$-disc by a disc labelled $w(s, t)$ together with additional $t$-arcs effecting the reduction from $w(t, t)$ to $w$; see Figure 4 . The arcs in the outer ring are $t$-arcs, but the legs inside are designated as $t$-legs or $s$-legs according to $w(s, t)$.
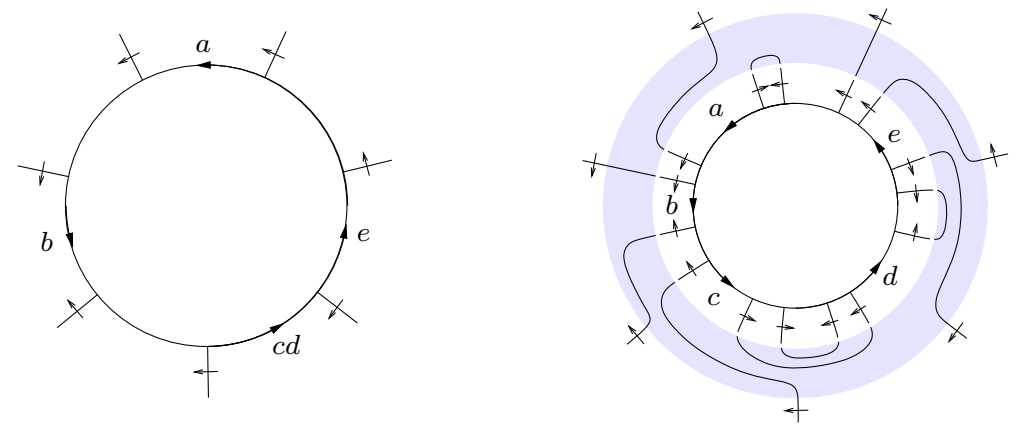

Figure 4: A $w$-disc with amenable word $w=a t t b t^{-1} t^{-1} c d t^{-1} e t^{2}$ and its blown up form $t\left(s^{-1} a s\right) t(b)\left(s^{-2} c s^{2}\right) t^{-1}\left(s^{-1} d s\right)\left(s^{-2} e s^{2}\right)$ inside

Next, we replace the $w(s, t)$-disc by a smaller $w(s, t)$-disc surrounded by "two-leg" discs which convert the $s$-legs to $t$-legs. A "two-leg" disc is labelled $h^{t}\left(h^{s}\right)^{-1}$ and actually has two legs labelled $t$, but two sets of legs labelled $s$ (corresponding to the occurrences of $s$ in $h^{s}$ and $h$ ). This is done as follows.

Consider the $s$-legs in a disc labelled by $w(s, t)$. They come in sets corresponding to the $x_{i}(s) \in H$. Now, $x_{i}(s)$ lies in $J$ and it follows that the $s$-legs can be paired off in nested cancelling pairs. Further, by counting 
the occurrences of $s$ (algebraically) from the start and considering a local minimum, we can find an adjacent cancelling pair of the form $s^{-1} s$. Deleting this pair and using induction, we see that the pairing off can be assumed to be coherent; in other words, cancelling pairs are always of this form (rather than $s s^{-1}$ ). We use induction on the number of such pairs. Consider an outermost pair. What lies inside also lies in $J$ and it follows that there is a two-leg disc which converts this pair into $t$-legs. By induction, we can find a set of two-leg discs to convert the remaining $s$-legs in similar cancelling pairs into $t$-legs. The effect is pictured in Figure 5 .

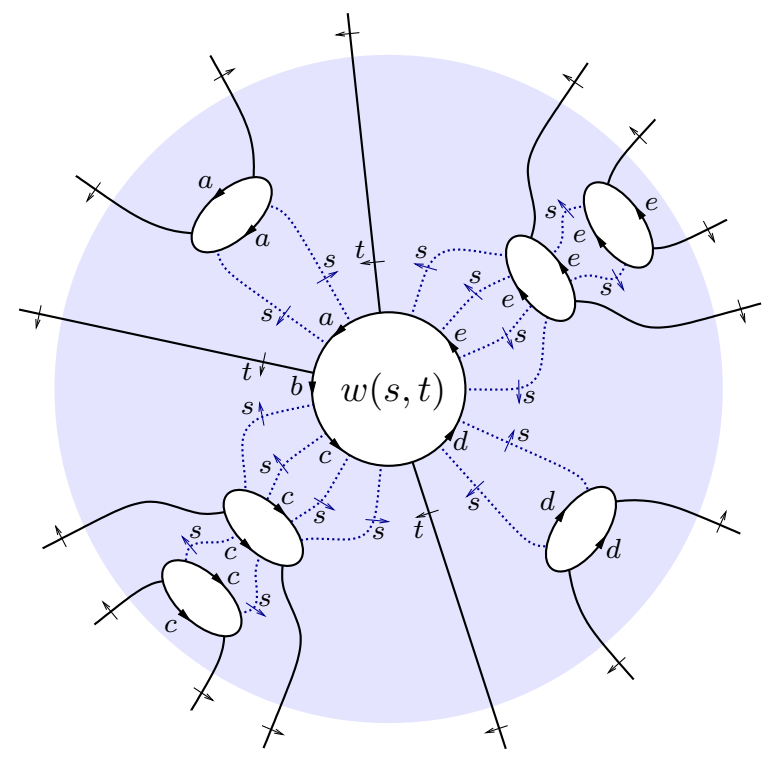

Figure 5: Converting $s$-legs to $t$-legs. The outer boundary reads $w(t, t)$.

Doing this for each $x_{i}$, we create a partial diagram having $t$-arcs and $s$-arcs, whose outer boundary reads $w(t, t)$. This diagram joins up with the outer ring of Figure 4.

The last step is to delete every $s$-arc and insert " $s$ " into the labels at its endpoints. Here, we are passing from a diagram over $G$ to a diagram over $\Gamma$. The result is a $W$-diagram; see Figure 6 . This $W$-diagram may not be irreducible because it may have strings of two-leg discs violating condition (4). Such a string can simply be replaced by a $t$-arc (cf [4, Figure 4, p. 134]). This has no effect on the group relations implicit in the diagram. This may result in floating $t$-circles which we now delete. The result is an irreducible $W$-diagram. Finally, note that every element of $Y$ (respectively 


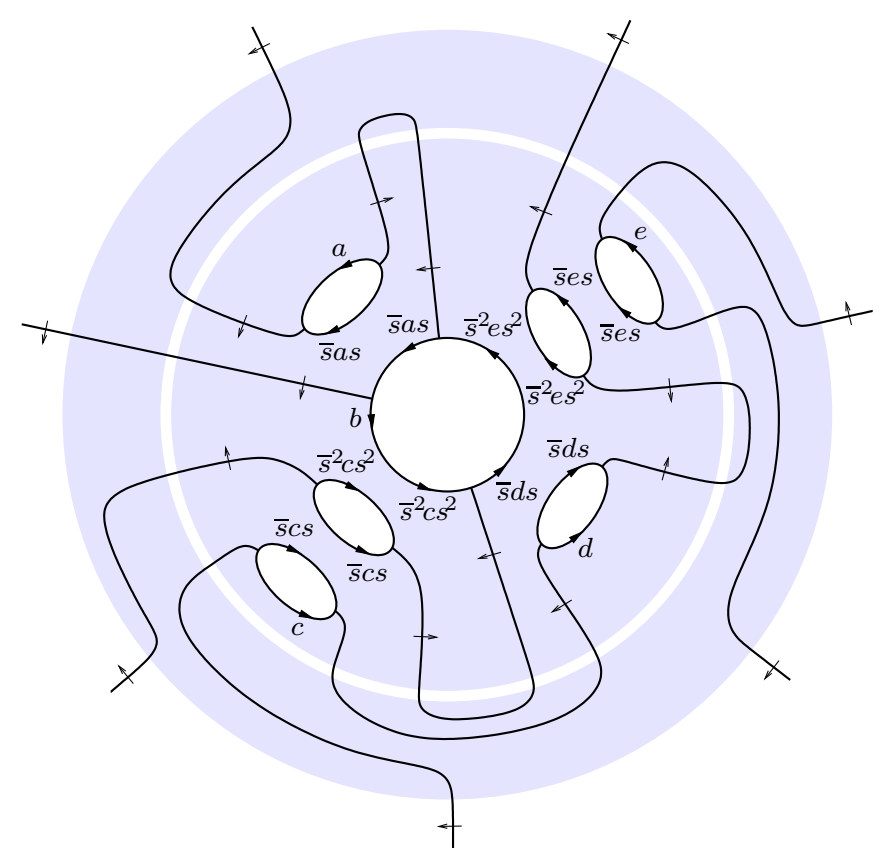

Figure 6: The $w$-disc with $t$-shape $t t t^{-1} t^{-1} t^{-1} t t$ realised as a $W$-diagram with $t$-shape $t t t^{-1}$ (the root shape of $t t t^{-1} t^{-1} t^{-1} t t$ )

$X$ ) is free relative to $H$ (respectively $H^{\prime}$ ), by [6, Lemma 4.3]. Thus, the diagram contradicts the Root Shape Theorem.

\section{Proof of the Main Theorem.}

We now return to the situation described in the Introduction. We are given a complex $K$ with torsion-free fundamental group, and $L=K \cup e^{1} \cup e^{2}$, with the 2-cell attached by an amenable word $w$. Let $K^{+}=K \cup e^{1}$.

First, observe that the Diagram Theorem translates directly into the statement that the map $\pi_{2}\left(K^{+}, K\right) \rightarrow \pi_{2}(L, K)$ is surjective. Using a standard transversality argument (as in [6, Section 3]), every element of $\pi_{2}(L, K)$ is represented by a $w$-diagram. If the diagram contains a disc labelled by $w$ or $\bar{w}$, then there must be a cancelling pair of such discs (as in Figure 2), by the Diagram Theorem. Such a pair can be removed by a homotopy. In this way, all discs labelled by $w$ or $\bar{w}$ can be removed. Then, the diagram represents an element of $\pi_{2}\left(K^{+}, K\right)$. 
The next lemma separates the Diagram Theorem into the adjunction problem (condition (a)) and an additional conclusion concerning $\pi_{2}$.

Lemma 3.1. Let $K \subset K^{+} \subset L$ be $C W$ complexes such that $K^{+}=K \cup$ $\{1$-cells $\}$ and $L=K^{+} \cup\{2$-cells $\}$. Then, $\pi_{2}\left(K^{+}, K\right) \rightarrow \pi_{2}(L, K)$ is surjective if and only if the following two conditions hold:

(a) $\pi_{1}(K) \rightarrow \pi_{1}(L)$ is injective,

(b) $\pi_{2}\left(K^{+}\right) \rightarrow \pi_{2}(L)$ is surjective.

Proof. The Lemma is easily deduced by inspecting the braid of long exact sequences for the triple $\left(L, K^{+}, K\right)$ :

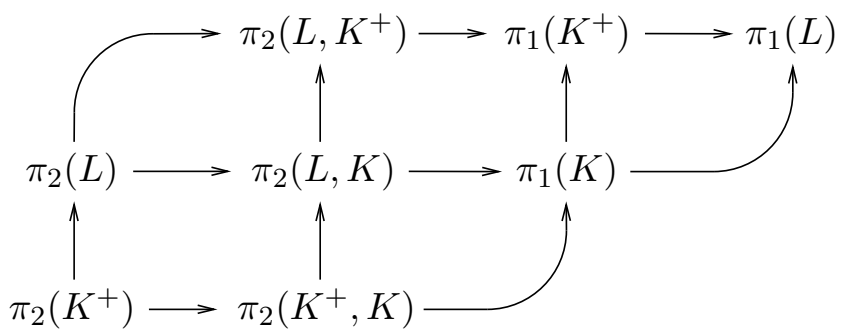

Apart from the braid, the only information needed is that the map $\pi_{1}(K) \rightarrow \pi_{1}\left(K^{+}\right)$is injective, which is clear from the hypotheses.

We also note the following basic result.

Proposition 3.2 ([3, Chapter III, 5.3]). Let $M$ be a $\mathbb{Z} G$-module whose underlying abelian group is a direct sum $\bigoplus_{i} M_{i}$. Suppose that the G-action preserves the direct sum decomposition and acts transitively on summands. Then, for any $i$, we have $M \cong \mathbb{Z} G \otimes_{\mathbb{Z} G_{i}} M_{i}$, where $G_{i}$ is the stabiliser of $M_{i}$.

Proposition 3.3. Assume the hypotheses of Lemma 3.1, and suppose the map $\pi_{2}\left(K^{+}, K\right) \rightarrow \pi_{2}(L, K)$ is surjective. Then, there is an isomorphism of $\mathbb{Z} \pi_{1}(L)$-modules $\pi_{2}(L) \cong \mathbb{Z} \pi_{1}(L) \otimes_{\mathbb{Z} \pi_{1}(K)} \pi_{2}(K)$.

This proposition (together with the Diagram Theorem) completes the proof of the Main Theorem.

Proof. Let $\widetilde{K}, \widetilde{K}^{+}$, and $\widetilde{L}$ be the universal covers of $K, K^{+}$, and $L$ respectively. Let $\widehat{K}^{+}$be the preimage of $K^{+}$in $\widetilde{L}$. Then $\widetilde{L}-\widehat{K}^{+}$consists of 2 -cells, 
and there are covering maps $\widetilde{K}^{+} \rightarrow \widehat{K}^{+} \rightarrow K^{+}$. Note that conditions (a) and (b) of Lemma 3.1 hold.

By condition (a), the preimage of $K$ in $\widetilde{L}$ consists of disjoint copies of $\widetilde{K}$. Then, $\widehat{K}^{+}$is this disjoint union, joined by 1-cells. The homology group $H_{2}\left(\widehat{K}^{+}\right)$is a direct sum of copies of $H_{2}(\widetilde{K})$, indexed by the copies of $\widetilde{K}$ in $\widehat{K}^{+}$. The group $\pi_{1}(L)$ acts on $\widehat{K}^{+}$by covering translations in $\widetilde{L}$, and the induced action on $H_{2}\left(\widehat{K}^{+}\right)$preserves the direct sum decomposition (and acts transitively on summands).

In the action of $\pi_{1}(L)$ on $\widehat{K}^{+}$, the stabilisers of the copies of $\widetilde{K}$ are the conjugates of $\pi_{1}(K)$ in $\pi_{1}(L)$. These subgroups are the stabilisers of the summands of $H_{2}\left(\widehat{K}^{+}\right)$as well. Choosing the appropriate copy of $\widetilde{K}$, Proposition 3.2 implies

$$
H_{2}\left(\widehat{K}^{+}\right) \cong \mathbb{Z} \pi_{1}(L) \otimes_{\mathbb{Z} \pi_{1}(K)} H_{2}(\widetilde{K}) .
$$

So far, we have used only condition (a). Now, consider the map $\pi_{2}\left(K^{+}\right) \rightarrow$ $\pi_{2}(L)$. In the following diagram, the lower vertical maps are Hurewicz homomorphisms, and the other maps are induced by inclusions or coverings.

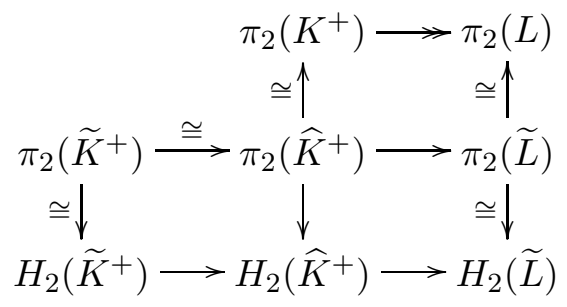

Condition (b) implies that the map $H_{2}\left(\widehat{K}^{+}\right) \rightarrow H_{2}(\widetilde{L})$ is surjective. It is injective because $H_{3}\left(\widetilde{L}, \widehat{K}^{+}\right)=0$, and therefore, there are isomorphisms $\pi_{2}(L) \cong H_{2}(\widetilde{L}) \cong H_{2}\left(\widehat{K}^{+}\right)$. The latter is isomorphic to $\mathbb{Z} \pi_{1}(L) \otimes_{\mathbb{Z} \pi_{1}(K)}$ $\pi_{2}(K)$.

\section{Counterexamples.}

In this section, we give examples showing that the Main Theorem can fail if either condition (1) or (2) is dropped. This is in contrast with the adjunction property which is expected to hold in much greater generality. The adjunction property holds for the first two examples below by [8] and [11]. Additional examples can be found in $[2,5,1,9,12]$.

Suppose that $L=K \cup e^{1} \cup e^{2}, K^{+}=K \cup e^{1}$, and $\pi_{1}(K) \rightarrow \pi_{1}(L)$ is injective. Keeping the notation of the previous section, we have $H_{2}\left(\widehat{K}^{+}\right) \cong$ 
$\mathbb{Z} \pi_{1}(L) \otimes_{\mathbb{Z} \pi_{1}(K)} \pi_{2}(K)$. We wish to construct examples where the injective map $H_{2}\left(\widehat{K}^{+}\right) \rightarrow H_{2}(\widetilde{L}) \cong \pi_{2}(L)$ is not surjective. For this, it suffices to show that $H_{2}(\widetilde{L}) \rightarrow H_{2}\left(\widetilde{L}, \widehat{K}^{+}\right)$is non-zero.

Any $w$-diagram defines a map $S^{2} \rightarrow L$ in which fibres of $t$-arcs map to $e^{1}$ and discs labelled $w$ or $\bar{w}$ map to $e^{2}$. Lifting this map to $\widetilde{L}$, one obtains a cycle representing an element of $H_{2}(\widetilde{L})$. The image of this cycle in $H_{2}\left(\widetilde{L}, \widehat{K}^{+}\right)$can be read off from the diagram, as a linear combination of 2-cells above $e^{2}$; see Figure 7. Note that the 2-cells above $e^{2}$ form a basis for $H_{2}\left(\widetilde{L}, \widehat{K}^{+}\right)$, and are acted on freely and transitively by $\pi_{1}(L)$. Hence, $H_{2}\left(\widetilde{L}, \widehat{K}^{+}\right) \cong \mathbb{Z} \pi_{1}(L)$. Given this relative 2 -cycle, one then needs to determine whether it is trivial, i.e. whether it cancels completely in $\mathbb{Z} \pi_{1}(L)$. To decide this, in general, one needs to be able to solve the word problem in $\pi_{1}(L)$.

First Example. This is the $w$-diagram shown in Figures 1 and 7 . The word $w=t a t b t^{-1} c$ has amenable $t$-shape, but the group $G$ has relations $b^{2}=c^{2}=$ $1, a^{-1} b a=c$ and so, is not torsion-free. Choose $K$ with fundamental group

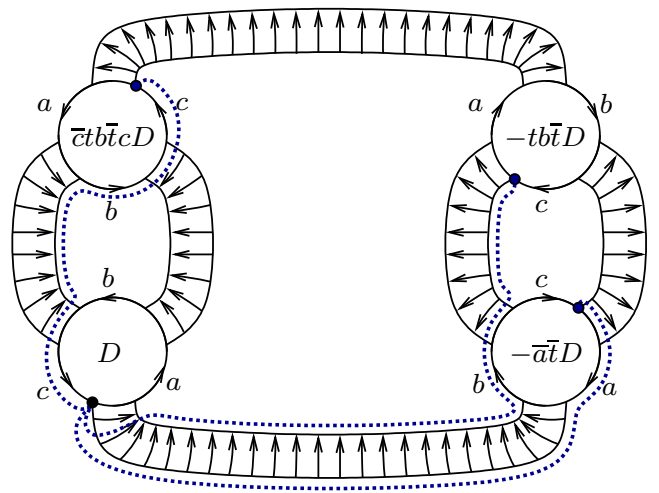

Figure 7: The diagram as an element of $H_{2}\left(\widetilde{L}, \widehat{K}^{+}\right)$

$G=\left\langle a, b, c \mid b^{2}=c^{2}=1, a^{-1} b a=c\right\rangle$ (the universal group of the diagram, in other words, the group with generators the coefficients of $w$ and relations read from the inside regions). Note that $\pi_{1}(K) \rightarrow \pi_{1}(L)$ is injective by [ 8 , Theorem 2], so the preceding discussion applies. The diagram represents a relative cycle of the form $D+\bar{c} t b \bar{t} c D-t b \bar{t} D-\bar{a} \bar{t} D$ in $H_{2}\left(\widetilde{L}, \widehat{K}^{+}\right)$. To see that this is non-trivial, it suffices to check that $t b \bar{t}$ and $\bar{a} \bar{t}$ are non-trivial in $\pi_{1}(L)$, for then the term $D$ does not cancel with anything. For this, we note that $\pi_{1}(L)=\langle G, t|$ tatbt $\left.^{-1} c\right\rangle$ acts as a reflection group in the plane. Take 
$\ell_{1}$ and $\ell_{2}$ to be lines meeting with angle $\pi / 3$, and let $a, b$, and $c$ be reflection across $\ell_{1}$, and $t$ reflection across $\ell_{2}$. Then $\bar{a} \bar{t}$ is an order 3 rotation and $t b \bar{t}$ a reflection, so both are non-trivial.

Relative presentations of the form $\left\langle G, t \mid t a t b t^{-1} c\right\rangle$ are explored fully in [5]. In this paper (building on results in [2]), a near-complete characterisation is given of groups that admit irreducible diagrams based on $w=t a t b t^{-1} c$.

Second Example. Let $w$ have $t$-shape $t^{n}$ for some $n>1$. As noted earlier, this $t$-shape is not amenable. Consider the $w$-diagram consisting of two discs, labelled $w$ and $\bar{w}$, with $t$-arcs joined cyclically. However, these $t$-arcs should be joined so that the diagram is irreducible, meaning that the two basepoints do not line up. The group relations implied by this diagram simply say that various coefficients are equal, in fact, that $w$ itself is a proper power. So, this irreducible diagram is valid over any group $G$. Note that the universal group of this diagram is in fact a free group.

To see that this diagram is non-zero in $H_{2}\left(\widetilde{L}, \widehat{K}^{+}\right)$, consider the map $G \rightarrow 1$ (the trivial group). This is realised by a map of $K$ to a point $K_{0}$ and $L$ to $L_{0}=e^{1} \cup_{n} e^{2}$ (the pseudo projective plane $\mathbb{P}_{n}$ with fundamental group $\mathbb{Z} / n \mathbb{Z})$. The diagram now represents a non-trivial element of the form $D-t^{k} D$ in $H_{2}\left(\widetilde{L}_{0}, \widehat{K}_{0}^{+}\right)$where $k$ is the relative shift between basepoints. In fact, this diagram, regarded as a map $S^{2} \rightarrow \mathbb{P}_{n}$, represents a standard generator of $\pi_{2}\left(\mathbb{P}_{n}\right) \cong \mathbb{Z}^{n-1}$, the others being obtained by varying $k$.

This example illustrates the difference between the notions of irreducibility used here and in [2], where this diagram would be considered reducible.

\subsection{Substitution.}

Before embarking on the third example, we remark that there is a very simple way to enlarge any diagram by substitution. Let $w \in G *\langle t\rangle$ be cyclically reduced and let $H$ be a group containing $G$. Let $u$ be any reduced word in $H *\langle t\rangle$ which starts and finishes with $t^{ \pm 1}$. Let $w^{\prime}$ be the word in $H *\langle t\rangle$ obtained by substituting $u^{\epsilon}$ for $t^{\epsilon}$ throughout. We want $w^{\prime}$ to also be cyclically reduced. In other words, we require that no cancellation between copies of $u$ is possible after substitution. To ensure this, we need to assume that if $u$ starts and finishes with $t^{\epsilon}, t^{-\epsilon}$, then all the coefficients of $w$ are non-trivial (i.e. middle coefficients as well as the top and bottom ones).

If $X$ is any $w$-diagram, then we can convert it to a $w^{\prime}$-diagram $X^{\prime}$ by replacing the $t$-arcs by $u$-arcs. In other words, we replace them by parallel 
sets of $t$-arcs corresponding to the occurrences of $t$ in $u$. The new regions contribute relations in $H$ of the form $x x^{-1}$ for the various coefficients $x$ of $u$. If $X$ is irreducible, then so is $X^{\prime}$; see Figure 8 .

Third Example Let $H \supset G$ be any groups, let $w \in G *\langle t\rangle$ have $t$-shape $t^{n}$ for some $n>1$ and let $u$ be any reduced word in $H *\langle t\rangle$ as above. Let $X$ be the $w$-diagram in the second example and $X^{\prime}$ the $w^{\prime}$-diagram obtained by substitution. The $t$-shape of $w^{\prime}$ is periodic and we can clearly obtain any periodic $t$-shape in this way.

A complete example of this construction is shown in Figure 8. This is the simplest case, namely $w=(a t)^{2}$ and $u=t b t^{-1}$.

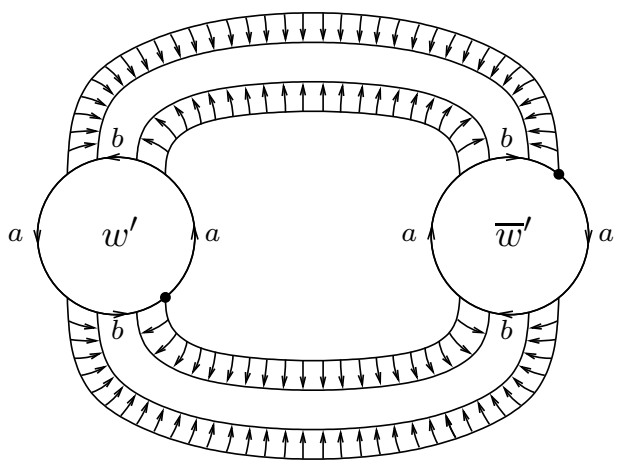

Figure 8: The result of substitution

The universal group for $X^{\prime}$ is again a free group. We cannot prove that $X^{\prime}$ is non-zero in $H_{2}\left(\widetilde{L}, \widehat{K}^{+}\right)$for all choices of this construction (and we suspect that it sometimes fails to be so), but for many choices we can prove this. In particular, if we take one of the coefficients $x$ of $u$ to generate a free factor of $H=\langle x\rangle * H_{0}$ and map $H_{0}$ to the trivial group and $t$ to 1 , then this example again maps to a generator of $H_{2}\left(\mathbb{P}_{n}\right)$ (with $x$ representing the 1-cell) as in the last example.

\subsection{Limits to Klyachko's methods.}

We have seen that Klyachko's methods imply the Diagram Theorem which in turn proves more than is necessary for the adjunction problem for torsionfree groups. We have also seen that this extra information is false for periodic $t$-shapes. This implies that Klyachko's methods will need modification if they are to prove the adjunction problem for periodic $t$-shapes. It should be 
noted that our examples where the group is torsion-free are reducible if one is allowed to change the basepoint on some $w$-discs when there is symmetry. Call an example in which this is not possible strongly irreducible. Thus, one necessary modification will be to incorporate an argument which makes strong irreducibility necessary.

It is possible to construct strongly irreducible examples, with power $t$ shape, with torsion in the universal group, which have the property that the torsion is not implied by any single region, but is a consequence of several. [The simplest example has $t$-shape $t^{3}$ and is based in the 1-skeleton of a cube. Labels are added to make the diagram irreducible. There are two ways of doing this up to obvious symmetries. The less symmetrical labelling is the required diagram.] The crash argument as presently formulated finds a single region where the torsion-free hypothesis is contradicted. So, if the methods are to work in general, they must also be extended (perhaps by extending the crash theorems) to provide a contradiction involving several regions.

We have not found an example of a strongly irreducible diagram over a torsion-free group.

Finally, it should also be noted that there are $t$-shapes which are neither periodic nor amenable. The simplest example is $t\left(t t^{-1}\right) t\left(t t^{-1}\right)^{2}$ and for such shapes, we have no information at all about either the adjunction problem or the Diagram Theorem.

\section{Further results.}

In [4], Cohen and Rourke proved that $g t$ is never in the normal closure $\langle\langle w\rangle\rangle$ of $w$ in $G *\langle t\rangle$. This result has an analogue for diagrams which generalises the Diagram Theorem. In [4, Section 6], the Cohen-Rourke result is extended to prove that if $w$ has amenable $t$-shape, then no word of $t$-shape $t^{n}$ is in $\langle\langle w\rangle\rangle$. Implicit in the proof of this is a stronger result which we shall deduce from the Extended Diagram Theorem, see the corollary below.

Let $G$ be a group and $w \in G *\langle t\rangle$. A $w$-diagram with boundary means a finite collection of discs in the standard 2-disc $D^{2}$ labelled by $w$ and $\bar{w}$ together with $t$-arcs which complete the legs compatibly with the orientations or which terminate at the boundary. The diagram cuts $D^{2}$ into a number of inside regions (which are disjoint from the boundary $S^{1}$ ) and a finite number of outside regions which meet the boundary. We require that the word in $G$ obtained by reading anticlockwise around each inside region is the identity in $G$. Reading round the part of the boundary of an outside 
region disjoint from $S^{1}$, we read a word in $G$ which we use to label the arc of intersection with $S^{1}$. We can now read a word $z \in G *\langle t\rangle$ from the labels on $S^{1}$ and the $t$-arcs arcs which terminate on $S^{1}$. We call $z$ the boundary word. Irreducibility has exactly the same meaning for diagrams with boundary as for ordinary diagrams.

Recall that the complexity of a $t$-shape is the number of Magnus differentiations required to reduce the shape to a power shape (ie $t^{q}$ for $q \in \mathbb{Z}$ ). The complexity of a word in $G *\langle t\rangle$ is the complexity of its $t$-shape.

Extended Diagram Theorem. Suppose that $w$ is a word in $G *\langle t\rangle$ with an amenable $t$-shape and that $G$ is a torsion-free group. Then, there are no irreducible $w$-diagrams with boundary a word of complexity strictly smaller than the complexity of $w$.

Corollary 5.1 (Extension of Cohen-Rourke Extension [4, p. 141]). Suppose that $w$ is a word in $G *\langle t\rangle$ with an amenable $t$-shape and that $G$ is a torsion-free group. Then, no word of complexity strictly smaller than the complexity of $w$ lies in the normal closure of $w$ in $G *\langle t\rangle$.

Notice that this result is sharp in the sense that there are obviously words of complexity the same as $w$ which do lie in $\langle\langle w\rangle\rangle$ (and obvious corresponding diagrams with boundary). Note also the analogy with small cancellation theory.

The proof of the Extended Diagram Theorem is a combination of the proof in [4, p. 141] and the proof of the Diagram Theorem in this paper. We first prove an Extended Root Shape Theorem, which is a version for $W$-diagrams where $W$ has the same meaning as in the Root Shape Theorem and the boundary is a word of $t$-shape $t^{q}$ for some $q \in \mathbb{Z}$. To prove this, we consider the cell subdivision of the 2 -sphere obtained by putting a vertex in each region and joining by edges across $t$-arcs. There is one outside cell $C$ which has $q$ edges crossing the $t$-arcs terminating on $S^{1}$. A traffic flow is defined as before with the car on the boundary of $C$ dealt with as described in [4, p. 141]: all the edges of $C$ are oriented the same way (without loss; let this be "uphill"). Notice that any other cell with an edge in common with $C$ has its car traverse that edge in the "downhill" direction, since adjacent cells induce opposite orientations on a common edge. Choose any point $\omega \in \partial C$ not at a vertex. The flow constructed as before for cells other than $C$ has the property that there are times when all cars are going uphill and hence are not on $\partial C$. This leaves time for car on $\partial C$ to rush round from just after $\omega$ to just before and hence there are no complete crashes on $\partial C$ except at $\omega$. 
There must be another crash and this leads to the identical contradiction as in the proof of the Root Shape Theorem.

To prove the Extended Diagram Theorem, we use the argument given in the proof of the ordinary Diagram Theorem to convert any $w$-diagram to a $W$-diagram: we convert the $w$-discs to partial $W$-diagrams exactly as illustrated in Figures 5 and 6 and we do the same for the boundary. Using the same notation as before, $w$ has complexity $m$. A word of complexity less than $m$ has root $t^{q}$ say and is obtained from $t^{q}$ by blowing up, i.e., by inserting elements of $J$. Each such element can be converted to an element of $G *\langle s\rangle$ by using 2-leg discs. This converts the boundary into a word of shape $t^{q}$. The final diagram contradicts the Extended Root Shape Theorem.

\section{References.}

[1] Y G Baik, W A Bogley, S J Pride, On the asphericity of length four relative group presentations, Internat. J. Algebra Comput. 7 (1997) 277312

[2] W A Bogley, S J Pride, Aspherical relative presentations, Proc. Edinburgh Math. Soc. 35 (1992) 1-39

[3] Kenneth S Brown, Cohomology of Groups, Springer-Verlag (1982)

[4] Marshall M Cohen, Colin Rourke, The surjectivity problem for onegenerator, one-relator extensions of torsion-free groups, Geom. Topol. 5 (2002) 127-142

[5] Martin Edjvet, On the asphericity of one-relator relative presentations, Proc. Roy. Soc. Edinburgh Sect. A 124 (1994) 713-728

[6] Roger Fenn, Colin Rourke, Klyachko's methods and the solution of equations over torsion-free groups, L'Enseignement Mathématique, 42 (1996) 49-74

[7] Roger Fenn, Colin Rourke, Characterisation of a class of equations with solutions over torsion-free groups, from: "The Epstein Birthday Schrift", (I Rivin, C Rourke and C Series, editors), Geometry and Topology Monographs, Volume 1 (1998) 159-166

[8] James Howie, The solution of length three equations over groups, Proc. Edinburgh Math. Soc. 26 (1983) 89-96 
[9] J Howie, V Metaftsis, On the asphericity of length five relative group presentations, Proc. London Math. Soc. (3) 82 (2001) 173-194

[10] Anton A Klyachko, A funny property of sphere and equations over groups, Comm. in Alg. 21 (1993) 2555-2575

[11] Frank Levin, Solutions of equations over groups, Bull. Amer. Math. Soc. 68 (1962) 603-604

[12] V Metaftsis, On the asphericity of relative group presentations of arbitrary length, Internat. J. Algebra Comput. 13 no. 3 (2003) 323-339

[13] Colin Rourke, On dunce hats and the Kervaire conjecture, Papers presented to Christopher Zeeman, University of Warwick (1988) 221-230, available from: http://www. maths. warwick.ac.uk/ cpr/ftp/dunce.ps

[14] H Short, Topological methods in group theory: the adjunction problem, Warwick Ph.D. thesis (1983)

[15] J H C Whitehead, On adding relations to homotopy groups, Ann. of Math. 42 (1941) 409-428

Mathematics Department

UNIVERSITY OF OKLAHOMA

NORMAN OK 73019

USA

E-mail address: forester@math.ou.edu

Mathematics Institute

UNIVERSITY OF WARWICK

Coventry, CV4 7AL

$\mathrm{UK}$

E-mail address: cpr@maths.warwick.ac.uk

ReCeived June 8, 2003. 